\title{
Commercial 4-Dimensional Echocardiography for Murine Heart Volumetric Evaluation after Myocardial Infarction
}

\section{Cody A Rutledge}

University of Pittsburgh

\section{George Cater}

University of Pittsburgh

\section{Brenda McMahon}

University of Pittsburgh

Lanping Guo

University of Pittsburgh

\section{Yijen Wu}

University of Pittsburgh

Flordeliza Villanueva

University of Pittsburgh

Brett A Kaufman ( $\nabla$ bkauf@pitt.edu )

University of Pittsburgh https://orcid.org/0000-0003-4767-4937

\section{Research}

Keywords: 4-dimensional ultrasound, preclinical echocardiography, mouse coronary artery ligation

Posted Date: December 10th, 2019

DOI: https://doi.org/10.21203/rs.2.18146/v1

License: (c) (i) This work is licensed under a Creative Commons Attribution 4.0 International License. Read Full License 


\section{Abstract}

Background: Traditional preclinical echocardiography $(\mathrm{ECHO})$ modalities, including 1-dimensional motion-mode (M-Mode) and 2-dimensional long axis (2D-US), rely on geometric and temporal assumptions about the heart for volumetric measurements. Surgical animal models, such as the mouse coronary artery ligation (CAL) model of myocardial infarction, result in morphologic changes that do not fit these geometric assumptions. New ECHO technology, including 4-dimensional ultrasound (4D-US), improves on these traditional models. This paper aims to compare commercially available 4D-US to Mmode and 2D-US in a mouse model of CAL.

Methods: 37 mice underwent CAL surgery, of which 32 survived to a 4 week post-operative time point. ECHO was completed at baseline, 1 week, and 4 weeks after CAL. M-mode, 2D-US, and 4D-US were taken at each time point and evaluated by two separate echocardiographers. At 4 weeks a subset of mice underwent cardiac magnetic resonance (CMR) imaging to serve as a reference standard. End systolic volume (ESV), end diastolic volume (EDV), and ejection fraction (EF) were compared between imaging modalities. Hearts were also collected for histologic evaluation of scar size and compared to wall motion severity index (WMSI).

Results: 4D-US provides closer agreement of ESV (Bias: -2.55\%) and EF (US Bias: 11.23\%) 4 weeks after CAL when compared to CMR, outperforming 2D-US and M-mode estimations. 4D-US is also more reliable than other modalities when estimating EDV ( $r=0.88)$, ESV ( $r=0.86)$ and EF ( $r=0.74)$ at 1 week when compared to 4 week imaging. 4D-US also allows for rapid assessment of WMSI, which correlates strongly with infarct size by histology $(r=0.77)$.

Conclusion: 4D-US outperforms M-Mode and 2D-US for volumetric analysis 4 weeks after CAL. 4D-US also provides more reliable measurements at 1 week after CAL and has less inter-user variability that other modalities. 4D-US allows for rapid calculation of WMSI, which correlates well with histologic scar size.

\section{Background}

Echocardiography $(\mathrm{ECHO})$ is a highly efficient and reliable tool for clinical and pre-clinical evaluation of cardiac function ${ }^{1-3}$. Advances in high-frequency and high-resolution image acquisition have allowed for increasing temporal and spatial resolution in small rodent models ${ }^{1,4,5}$. However, traditional ECHO modalities for evaluating ventricular size and function, including 1-dimensional motion-mode (M-mode) and 2-dimensional (2D-US) long-axis analysis, make geometric assumptions about the heart that limit their accuracy, particularly in the setting of heart disease, where regional ventricular shape abnormalities may exist ${ }^{6-8}$. For example, mouse models of cardiac pathology, particularly myocardial infarction (MI), frequently result in abnormal ventricular remodeling that deviates from the geometric assumption that the left ventricle has an ellipsoid shape, limiting the utility of conventional echocardiography in such settings and requiring careful consideration of modality choice ${ }^{9,10}$. 
Three-dimensional (3D) ECHO has gained favor recently in clinical and pre-clinical models, allowing full volume visualization of the heart by stacking concentric short-axis images to form a 3D representation at static time points ${ }^{4,11,12}$. 3D ECHO has been further improved by combining respiratory- and ECG-gated image acquisition to create 3D images throughout the cardiac cycle, referred to as 4-dimensional ultrasound (4D-US) ${ }^{13,14}$. 4D-US has recently became commercially available for pre-clinical models and has already been validated against cardiac magnetic resonance (CMR) imaging in a mouse model of hypertrophy ${ }^{15}$. While CMR imaging remains the gold standard for assessment of cardiac function in mice ${ }^{16-18}$, recent studies in 3D ECHO and 4D-US have shown considerable advances in scanning time and reliability ${ }^{13-15}$.

In this study, we aimed to validate commercially available 4D-US in a mouse model of myocardial infarction. We performed coronary artery ligation (CAL) surgery on mice to evaluate traditional M-mode, 2D-US, and 4D-US using the Vevo 3100 preclinical imaging system against CMR imaging. When compared to CMR, 4D-US outperforms M-mode and 2D-US in the evaluation of volumetric parameters 4 weeks after CAL. Additionally, 4D-US allows for reliable prediction of scar size using wall motion severity index (WMSI) compared to histologic analysis. Finally, 4D-US is superior to other modalities when comparing images collected at 1 week after CAL to those collected at 4 weeks, suggesting increased reliability early after CAL.

\section{Methods}

\section{Coronary Artery Ligation and Mouse Model}

All animal use was performed at the University of Pittsburgh in compliance with the National Institutes of Health Guide for Care and Use of Experimental Animals and was approved by the University of Pittsburgh Animal Care and Use Committee. Previously characterized mice harboring a floxed TFAM gene via loxstop-lox manipulation were crossed with a-myosin heavy chain Cre transgene (MHC-Cre) to generate mice that were MHC-Cre (+) $x$ LSL-TFAM and MHC-Cre (-) $x$ LSL-TFAM. Both males and females were used in this study. Mice were aged to twelve weeks, at which point they underwent baseline echocardiography followed by coronary artery ligation surgery. Mice were anesthetized and ventilated prior to open thoracotomy through the 4th rib followed by opening of the pericardium and coronary artery ligation (CAL) by suture placement around the proximal coronary artery ${ }^{27}$. All surviving underwent follow-up echocardiography at 1 week and 4 weeks. A total of 33-mice were evaluated.

\section{Echocardiography}

Mice were anesthetized using isoflurane delivered by nose cone at baseline, 1 week following CAL, and 4 weeks following CAL. Depilatory cream was applied to the thorax to remove hair. Animals were maintained at $37^{\circ} \mathrm{C}$ via heating pad and rectal probe and monitored using surface ECG limb electrodes throughout imaging. Transthoracic echocardiography was performed using the Vevo 3100 imaging 
systems (Visual Sonics, Toronto, Canada) with a $40 \mathrm{MHz}$ linear probe attached to a step motor. Heart rate was maintained between $400-500 \mathrm{bpm}$ during imaging by adjusting isoflurane concentration. M-mode and B-mode images of the heart were obtained for at least ten cardiac cycles in the parasternal long axis and mid-papillary muscle level short axis views. For 4D image acquisition, the step motor was positioned just below the apex and the motor aligned to take concentric short axis images in $0.2 \mathrm{~mm}$ steps. At each position, a complete cardiac cycle was recorded using automated ECG and respiratory gating. 4D images were constructed using commercially available Vevo 4D image software. Image analysis was performed independently by two blinded sonographers (CR and BM). End-diastolic volume (EDV), End-systolic volume (ESV), Ejection Fraction (EF), and Stroke Volume (SV) were calculated using the following formulas:

M-Mode (short-axis):

$E D V=\left[7.0 /(2.4+\right.$ LVID;d $) \times$ LVID; [3 $\left.^{3}\right]$ where LVID;d = Left ventricular internal diameter at end diastole ESV $=\left[7.0 /\left(2.4+\right.\right.$ LVID;s) $\times$ LVID;s $\left.{ }^{3}\right]$ where LVID;s = Left ventricular internal diameter at end systole $\mathrm{EF}=100 \times((E D V-E S V) / E D V)$

2D-US analysis of the parasternal long-axis was completed using operator-defined LV trace function of Vevo software and calculations of EDV, ESV, and EF made by Simpson's method. 4D-US measurements were calculated directly from volumetric measurements based on operator-defined edge-tracing using Vevo 4D imaging software.

\section{WMSI}

WMSI was calculated using a 16-segment model collected from 3 short axis views collected during 4D-US image acquisition. Short axis views were obtained $1 \mathrm{~mm}, 3 \mathrm{~mm}$, and $5 \mathrm{~mm}$ from the apex to allow for standardization between animals. The most distal image was divided into 4 sections and the remaining sections divided into 6 sections as previously described (Fig. 4) ${ }^{24,25}$. Individual sections were graded as: 1- normal, 2- hypokinetic, 3- akinetic, 4- dyskinetic, 5- aneurysmal. WMSI was calculated as sum off all motion scores over 16.

\section{CMR}

Mice were anesthetized with $4 \%$ isoflurane mixed with room air in an induction box for 1 to 3 minutes. The depth of anesthesia was monitored by toe reflex, extension of limbs, and spine positioning. Once anesthetized, the mouse was placed in a neonatal mouse holder and the anesthesia was maintained by 1.5 to $2 \%$ isoflurane and $100 \%$ oxygen via a nose cone. Respiration waveforms were continuously monitored using a small pneumatic pillow under the animal's diaphragm connected to a magnetcompatible pressure transducer (SA Instruments, Stony Brook, NY). CMR was performed on a Bruker Biospec 7T/30 system (Bruker Biospin MRI, Billerica, MA) with a 35-mm quadrature coil for both transmission and reception. The Bruker Intragate module was used for image-gated cine MRI with 
retrospective navigation. Subcutaneous injection of Multi-Hance (Gadobenate dimeglumine, $529 \mathrm{mg} / \mathrm{ml}$, Bracco Diagnostics, Inc, Monroe Twp, NJ 08831) was administered immediately before the CMR acquisition at $0.1 \mathrm{mmol} \mathrm{Gd} / \mathrm{kg}$ bodyweight. $T_{1}$-weighted images to highlight LGE were acquired $15-20$ minutes after the subcutaneous administration of Multi Hance. Eight $\mathrm{T}_{1}$-weighted short-axis imaging planes covering the whole ventricular volume with no gaps were acquired with the following parameters: Field of view $(F O V)=2.5 \mathrm{~cm} \times 2.5 \mathrm{~cm}$, slice thickness $=1 \mathrm{~mm}$, in-plane resolution $=0.97 \propto \mathrm{m}$, flip angle $(F A)=10$ degrees, echo time $(T E)=3.059 \mathrm{msec}$, repetition time $(T R)=5.653 \mathrm{msec}$. White-blood cine movies with 20 cardiac phases were acquired for each mouse with equivalent temporal resolution for the cine loops was about 16.5-21.5 ms per frame. Eight short-axis imaging planes covering the whole ventricular volume with no gaps and one long-axis plane were acquired with the following parameters: Field of view $(F O V)=2.5 \mathrm{~cm} \times 2.5 \mathrm{~cm}$, slice thickness $=1 \mathrm{~mm}$, in-plane resolution $=0.97 \propto \mathrm{m}$, flip angle $(F A)=30$ degrees, echo time $(T E)=1.872 \mathrm{msec}$, repetition time $(T R)=38.293 \mathrm{msec}$.

The extent of myocardial infarction was defined by the percentage of the myocardium displaying hyperintensity 15-20 minutes after $\mathrm{Gd}$ administration. To obtain the proportion of myocardial infarction, the area of hyperintensity was manually traced by a blinded operator on the Paravision 5.1 Xtip software (Bruker Biospin MRI, Billerica, MA). The extent of myocardial hemorrhage was defined by dark hypointensity on the cine images. To obtain the proportion of myocardial hemorrhage, the area of hypointensity was manually traced by a blinded operator on the Paravision 5.1 Xtip software (Bruker Biospin MRI, Billerica, MA). The left ventricular endocardium and epicardium boundaries of each imaging slice at the end-systole (ES) and the diastole (ED) were manually traced by a blinded operator on the Paravision 5.1 Xtip software (Bruker Biospin MRI, Billerica, MA) to calculate the following functional parameters: left ventricular blood volume (LVV), left ventricular wall volume (LV wall), LV mass, stroke volume (SV), ejection fraction (EF), heart rate (HR), cardiac output (CO), longitudinal shortening, and radial shortening. LVV is calculated by summation of all the short-axis slices. The ejection fraction (EF) was calculated using the following equation:

$E F=\frac{\sum_{i} A_{i}^{e s} h_{i}}{\sum_{i} A_{i}^{e d} h_{i}} \times 100 \%$,

where

$A_{i}^{e s}$

is the internal left ventricle area of slice $i$ at end systole,

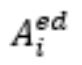

is the internal left ventricle area of slice $i$ at end diastole, and $h_{i}$ is the thickness of each scanned slice.

\section{Tissue Histology}


LV tissue was fixed overnight in $10 \%$ formaldehyde at $4{ }^{\circ} \mathrm{C}$. Tissues were then washed with PBS and transferred to $70 \% \mathrm{EtOH}$ and stored at room temperature. After fixation, tissues were brought to the Histology Core at the University of Pittsburgh and sectioned into $10 \mu \mathrm{m}$ slices at $1 \mathrm{~mm}$ intervals throughout the myocardium. Sections were stained with hematoxylin and eosin (H\&E) and Trichrome stains and images obtained on a TissueFAXS Histo (TissueGnostics, Vienna, Austria) upright brightfield microscope and images obtained by HistoQuest software. Image analysis was performed by automated red and blue channel separation using Image Measurement 9.0 (Bersoft Imaging, Cologne, Germany).

\section{Statistical Analysis}

Differences between imaging modalities in Fig. 1 were evaluated by Bland-Altman analysis and are expressed as $\%$ bias and $95 \%$ level of agreement (LOA). Bland-Altman percentage bias was calculated as (100*(B-A)/Average vs Average). Intraclass correlation was used to evaluate correlation between two users (CR and BM). Regression analysis was performed using Spearman rank correlation to compare scar size based on histology to WMSI and CMR computations (Fig. 4) and by Pearson correlation to compare week 1 to week 4 volumetric measurements (Fig. 5). Correlation was graded as poor (0.0-0.5), moderate (0.5-0.7), strong (0.7 to 0.9$)$, or very strong (0.9-1.0). Supplemental Fig. 2 data are expressed and mean \pm standard error. $p \leq 0.05$ was considered significant for all comparisons All statistical analysis was completed using Graphpad Prism 7 software (San Diego, CA).

\section{Results}

\section{Animal Model of Myocardial Infarction}

In order to model myocardial infarction, thirty-seven male mice underwent CAL surgery at 8 weeks of age. Thirty-two of these mice survived to 4 weeks post-CAL (86.4\%, Table 1). The mouse background was MHC-CRE (+) x LSL-TFAM or MHC-CRE (-) x LSL-TFAM. Subgroup analysis was performed between MHC $(+)(n=22)$ and MHC $(-)(n=15)$ groups and there was no difference noted in survival, scar size, baseline end-diastolic volume (EDV), end-systolic volume (ESV), and ejection fraction (EF), as well as 4 week EDV, ESV, or EF between groups by any ECHO modality, allowing pooled sampling of transgenic mice (Supplemental Fig. 2, Supplemental Table 1). 
Table 1

Baseline and 4 week post CAL Animal Data

\begin{tabular}{|lll|}
\hline & Total Mice & Percent \\
\hline Survival & $32 / 37$ & $86.40 \%$ \\
\hline Maseline Weight (g) & 25.26 & 0.58 \\
\hline Week 4 Weight (g) & 25.44 & 0.537 \\
\hline Baseline HR (bpm) & 454.96 & 7.96 \\
\hline Week 4 HR (bpm) & 482.18 & 15.25 \\
\hline & Mean \pm SEM & Range \\
\hline Histologic Scar Size & $21.54 \pm 4.07 \%$ & $4.02 \%-57.0 \%$ \\
\hline WMSI & $1.32 \pm 0.05$ & $1.065-2.0625$ \\
\hline CMR Hyperintense Volume & $13.53 \pm 2.22 \%$ & $5.52-28.10 \%$ \\
\hline
\end{tabular}

\section{Comparison of ECHO Modalities to CMR}

To compare imaging modalities following infarction, mice were evaluated by $\mathrm{ECHO}$ using traditional Mmode, 2D-US, and 4D-US (representative images in Fig. 1, mean values reported in Table 2, $n=32$ ) at baseline, 1 week following CAL, and 4 weeks following CAL. One-day after the 4 week ECHO was performed, a subset of randomly chosen mice underwent CMR to serve as reference standard for volumetric measurements (Fig. 1, $n=12$ ). Bland-Altman analysis of M-mode, 2D-US, and 4D-US was used to compare the percentage difference of ECHO modalities to CMR (Fig. 2). Of the three ECHO modalities, 4D-US demonstrated the lowest bias when comparing ESV (4D-US Bias: 2.55\% LOA: -61.55 to 66.66, 2DUS Bias: $29.84 \%$ LOA: -43.10 to 102.8, M-Mode Bias: 43.57\% LOA: -21.87 to 109.0; Fig. 2) and EF (4D-US Bias: $-11.23 \%$ LOA: -56.26 to 33.80 , 2D-US Bias: $-24.42 \%$ LOA: -87.99 to 39.16 , M-Mode Bias: $-12.90 \%$ LOA: -53.80 to 28.00 ) at 4 weeks. 4D-US was outperformed by 2D-US when evaluating EDV (4D-US Bias: $-13.21 \%$ LOA: -47.71 to 21.28 , 2D-US Bias: $5.84 \%$ LOA: -24.47 to 36.15 , M-Mode Bias: $35.17 \%$ LOA: 0.73 to 69.61). Linear regression was also performed between ECHO modalities and CMR and demonstrates strong to very strong correlation between 4D-US and CMR (EDV: $r=0.887, E S V: r=0.943, E F: r=0.811$; Supplemental Fig. 1). 2D-US has lower correlation values for every modality (EDV: $r=0.806, E S V: r=$ 0.793 , EF: $r=0.406$ ). M-Mode correlation is slightly higher than 4D-US for EDV, but lower for ESV and EF (EDV: $r=0.902$, ESV: $r=0.882$, EF: $r=0.747$ ). Inter-user variability was evaluated between two users (CR and $\mathrm{BM}$ ) for each modality by calculating the intraclass correlation (ICC) of measurements. 4D-US demonstrated the highest ICC for estimation of EDV (4D-US: 0.91, 2D-US: 0.72, M-Mode: 0.80) and ESV 
(4D-US: 0.93, 2D-US: 0.72, M-Mode: 0.79). M-mode had a higher ICC for evaluation EF (4D-US: 0.64, 2DUS: 0.31, M-Mode: 0.69).

Table 2. Mean Volumetric Data at 1 and 4 weeks by Imaging Modality

\begin{tabular}{|lllll|}
\hline 1 week & MRI & 4D-US & 2D-US & M-mode \\
\hline EF $(\%)$ & - & $43.94 \pm 2.98$ & $38.83 \pm 2.28$ & $48.47 \pm 3.26$ \\
\hline SV $(\mu \mathrm{L})$ & - & $16.37 \pm 1.38$ & $22.54 \pm 1.31$ & $36.54 \pm 2.03$ \\
\hline ESV $(\mu \mathrm{L})$ & - & $23.88 \pm 3.77$ & $40.12 \pm 4.84$ & $42.16 \pm 6.97$ \\
\hline EDV $(\mu \mathrm{L})$ & - & $40.25 \pm 4.30$ & $62.66 \pm 5.516$ & $94.45 \pm 7.43$ \\
\hline 4 week & MRI & 4D-US & 2D-US & M-mode \\
\hline EF $(\%)$ & $45.89 \pm 5.66$ & $38.89 \pm 3.41$ & $34.27 \pm 3.14$ & $40.04 \pm 4.43$ \\
\hline SV $(\mu \mathrm{L})$ & $27.11 \pm 1.46$ & $21.41 \pm 1.60$ & $21.76 \pm 1.12$ & $33.28 \pm 3.21$ \\
\hline ESV $(\mu \mathrm{L})$ & $41.75 \pm 9.57$ & $37.72 \pm 6.05$ & $51.44 \pm 9.99$ & $57.66 \pm 9.45$ \\
\hline EDV $(\mu \mathrm{L})$ & $68.86 \pm 8.96$ & $59.13 \pm 6.66$ & $73.20 \pm 10.16$ & $94.34 \pm 7.82$ \\
\hline
\end{tabular}

\section{Wall Motion Severity Index (WMSI) and Scar Sizing}

In order to quantify scar size following CAL, we utilized gadolinium-enhanced CMR, step-wise short-axis images obtained via 4D-US and trichrome staining of tissue sections, which was used as the gold standard (Fig. 3, $n=12$ ). Following sacrifice 4 weeks after CAL, a subset of these mice underwent histologic staining to evaluate scar size. Scar size was estimated histologically by comparing the pixel area of blue stained fibrotic tissue to red stained viable tissue on trichrome-stained cross sections taken every $1 \mathrm{~mm}$ (Table 1, Fig. 3, $\mathrm{n}=16$ ). The mean scar volume was $21.54 \pm 4.07 \%$ (Range $4.02-57.00 \%, n=$ 16, Table 1). Late gadolinium-enhanced CMR images were used to evaluate hyperintense tissue/LV area, which yielded a mean volume of $13.53 \pm 2.22 \%$ (range $5.52-28.10 \%$, Table 2 ). WMSI was calculated across 16 sections of myocardium, with average WMSI $1.32 \pm 0.05$ (range 1.07-2.07, Table 2, $n=32$ ). Spearman rank correlation was used to compare both WMSI and CMR hyperintense regions/LV area to histologic staining (Fig. 4). WMSI correlates strongly with histologic scar size $(r=0.77, p=0.008)$ and CMR hyperintense volume correlates very strongly with scar size $(r=0.90, p=0.0003)$.

\section{Comparison of Week 1 and Week 4 Data}

We next evaluated how well traditional M-mode, 2D-US, and 4D-US correlate between week 1 and week 4 following CAL in order evaluate the validity of each modality early in the recovery stage. Image quality tends to be reduced early after CAL on account of residual air in the chest and local inflammation at the 
thoracotomy site. 4D-US, 2D-US, and M-mode images were taken at Week 1 and Week 4 following CAL. Week 1 and Week 4 images of the same modality were compared to each other using Pearson correlation. 4D-US demonstrates strong correlation between time points in the evaluation of EDV $(r=0.88$, $p<0.0001$, Fig. 5), ESV $(r=0.86, p<0.00001)$, and EF $(r=0.74, r<0.0001)$. 2D-US provided strong correlation in EDV $(r=0.81, p<0.0001)$ and ESV $(r=0.86, p<0.0001)$ and moderate correlation of EF $(r=$ $0.52, p<0.01)$. M-mode demonstrated strong correlation between week 1 and week 4 EDV $(r=0.75, p<$ $0.001)$ and moderate correlation between $\operatorname{ESV}(r=0.63, p<0.001)$ and $\operatorname{EF}(r=0.64, p<0.01)$.

\section{Discussion}

This study evaluated commercially available 4D-US in a mouse model of myocardial infarction 1 week and 4 weeks after CAL and compared 4D-US endpoints to 2D-US, M-mode, CMR, and histologic parameters. The mouse CAL model presents a challenge for evaluation using traditional echocardiography, given that wall motion abnormalities and dyssynchronous contraction can cause abnormal LV geometry. Traditional M-mode and 2D-US assume ellipsoid geometry for volumetric measurements ${ }^{6}$. Following CAL, mice typically have a hyperdynamic base, static apex, and increased sphericity that limit the effectiveness of the geometric assumptions ${ }^{17,19-21}$. 4D-US allows for real-time image analysis at multiple planes encompassing the LV that is gated by ECG and respiration ${ }^{13,14}$ to minimize motion artifact during image reconstruction The result is a data-driven volumetric model that eliminates spatial and temporal assumptions about LV morphology. Our data demonstrate that for calculation of EF and ESV, 4D-US provides closer agreement when compared to benchmark CMR than other ECHO modalities 4 weeks after CAL. Additionally, we show that 4D-US has lower inter-user variabilty when measuring ventricular volumes at 4 weeks. 4D-US has the highest correlation when comparing volumetric measurements taken at 1 week to those taken at 4 week when compared to other $\mathrm{ECHO}$ modalities. Finally, 4D-US allows for simplified evaluation of WMSI, which correlates well with scar size by histologic analysis.

We find that 4D-US has lower percentage bias by Bland-Altman analysis than 2D-US and M-mode compared to CMR for the evaluation of ESV and EF at 4 weeks (Fig. 2), suggesting more accurate assessment of volumetric parameters. 4D-US is outperformed by 2D-US in the evaluation of EDV at this time point. However, across the three endpoints evaluated (EDV, ESV, and EF), 4D-US is the most reliable modality in terms of bias and $95 \%$ level of agreement. 4D-US has much lower bias than M-mode, which demonstrated $>40 \%$ bias in the estimation of ESV. 2D-US bias was improved compared to M-mode, but is characterized by the highest inter-user variability as demonstrated by lowest ICC for the evaluation of EDV, ESV, and EF (Fig. 2C). Inter-user variabilty is lowest using 4D-US for evaluation of EDV and ESV, but was mildly outperformed by M-Mode when evaluating EF (Fig. 2C). In total, our data is consistent with other studies that finds that 4D-US provides more accuracy and less inter-user variability when comparing to CMR than 2D-US or M-mode values, making it the optimal modality for surgical animal studies.

4D-US uniformly underestimates EDV and ESV when compared to CMR, though the changes were more pronounced in EDV (Fig. 2B, Table 2). The 4D-US measurements may suffer limitations in surgical mouse 
models, as the base of the heart is difficult to clearly visualize given the overlying scar following thoracotomy required in the CAL surgery, and may result in lower total volumes. However, a similar underestimation of volumes was previously noted in evaluation of round "phantoms" using identical software ${ }^{15}$, suggesting that a systemic underestimation of volumes may be present in this modality. Regardless of this underestimation, the modality still represents an improvement over other traditional ECHO modes.

Recent work by Russo et al. has shown that step-wise short-axis ECHO imaging performed on CAL mice strongly correlates with $\mathrm{CMR}^{17}$, providing reproducible volumetric evaluations nearly on par with $\mathrm{CMR}$, but at a fraction of the time and cost required for CMR. Our data support the increased value of this step-wise approach, but incorporate ECG- and respiratory-gating and the higher resolution of the Vevo-3100 for enhanced temporal and spatial resoluation. Soepriatna et al. previously demonstrated similar benefits of gated 4D-US in infarcted mice using manual 3-D reconstruction throughout the cardiac cycle ${ }^{14}$. While that study excellently demonstrated the value of the hardware and imaging through the cardiac cycle, here we use an easily-accessible commercial system, which utilizes edge-tracing software to simplify volumetric calculation for users, resulting in both rapid analysis and low inter-user variability.

An additional benefit of the 4D-US imaging modality is the ability to quickly and reproducibly quantify scar characteristics using clinical scales of wall-motion. Previous works have quantified scar size using ECHO based on wall-motion abnormalities from 2D-US and 3-d reconstruction, demonstrating good comparison between ECHO estimations and histologic scar size ${ }^{22,23}$. We evaluated WMSI using a 16segment model across three short axis views used in the clinical settings as previously described ${ }^{24,25}$. We found that WMSI correlates strongly with histologic analysis of scar size $(r=0.77$, Fig. 5), while gadolinium-enhanced CMR correlated very strongly $(r=0.90)$. The 4D-US method allows for easy standardization on the WMSI across animals, as the automated $0.2 \mathrm{~mm}$ step function utilized in this paper can be utilized to standardize the height of short-axis image. In this study, we used three short-axis images taken $1 \mathrm{~mm}, 3 \mathrm{~mm}$, and $5 \mathrm{~mm}$ from the apex to quantify WMSI. It should be noted that his model utilizes permanent coronary occlusion. Models of transient occlusion may be complicated by myocardial stunning, which may impair the value of WMSI and its correlation to scar size.

A key point of this study is the comparison of 1 week and 4 weeks with 4D-US, 2D-US, and M-mode to assess the predictive value of early imaging vs 4 week imaging. A major challenge following CAL is that cardiac remodeling is a dynamic process, characterized by progressive LV dilation, scar thinning, and compensatory hypertrophy of viable tissue ${ }^{9,19,26}$. Early imaging after CAL in the mouse model is limited by free air in the chest cavity and inflammation at the thoracotomy site that reduces the image quality of ECHO images. These post-surgical changes compound the limitations of 2D-US and M-Mode, as slight errors in 1-dimensional or 2-dimensional analysis cause major deviations in volumetric calculations. 4DUS does not rely on these geometric expansions. We find that 4D-US has highest correlation across volumetric measurements when comparing 1 week and 4 week values. 


\section{Potential caveats}

A potential confounder in the study is the use of multiple genotypes on a single background. Specifically, we utilized transgenic mice for the evaluation of volumetric measurements, pooling data from a-MHC-Cre $(+) \times$ TFAM-flox and a-MHC-Cre (-) x TFAM-flox mice. Because we found no difference between survival, scar size, baseline and 4 week EDV, ESV, or EF between groups (Supplemental Fig. 1, Supplemental Table 1), we included both models for this data set. We believe that the transgenic background does not limit the correlations drawn in this paper between volumetric studies and agrees with the NIH's effort to reduce animal suffering by utilizing mice readily available in our lab.

\section{Conclusions}

In this paper we demonstrate that commercially available 4D-US provides quick and reliable volumetric measurements of the heart following CAL that compares favorably to CMR values. 4D-US correlates better with CMR than 2D-US and M-Mode for evaluation of volumetric parameters 4 weeks after CAL. 4DUS also allows simple evaluation of WMSI, which is a clinically relevant metric that correlates well with scar size by histologic analysis. Finally, 4D-US provides more reliable volumetric assessment early after CAL compared to 2D-US and M-mode assessments.

\section{Declarations}

\section{Ethics approval and consent to participate}

All animal use was performed at the University of Pittsburgh in compliance with the National Institutes of Health Guide for Care and Use of Experimental Animals and was approved by the University of Pittsburgh Animal Care and Use Committee

\section{Consent for publication}

Not Applicable

\section{Availability of data and materials}

The datasets used and analyzed during the current study are available from the corresponding author on reasonable request.

\section{Competing interests}

The authors declare that they have no competing interests.

\section{Funding}

Research reported in this manuscript was supported by: American Heart Association Transformation Project Award 18TPA34230048, NIH Instrument Grant for Advanced High-Resolution Rodent Ultrasound 
Imaging System 1S100D023684-01A1.

\section{Authors' contributions}

CR obtained, analyzed and interpreted ECHO data was a major contributor to writing the manuscript. GC and FV performed manuscript review and helped write the discussion portion. BM contributed to ECHO analysis. LG performed all animal surgeries. BK is the primary investigator overseeing the project.

\section{Acknowledgements}

We would like to thank Dr. Andrew Althouse for guidance of statistical analyses.

\section{List Of Abbreviations}

2-Dimensional Ultrasound 2D-US

3-Dimensional

3D

4-Dimensional Ultrasound 4D-US

Anterior

A

Anterior Lateral

AL

Anterior Septal

AS

Cardiac Magnetic Resonance

CMR

Cardiac Output

CO

Coronary Artery Ligation

CAL

$\mathrm{ECHO}$

Echocardiography

Echo Time

TE

Ejection Fraction

$\mathrm{EF}$

End Diastole

ED

End Diastolic Volume

EDV

End Systole

ES

End Systolic Volume

ESV

Field of View

FOV 
Flip Angle

$\begin{array}{ll}\text { Hematoxylin and Eosin } & \text { H\&E } \\ \text { Inferior } & \text { I } \\ \text { Inferior Lateral } & \text { IS } \\ \text { Inferior Septal } & \text { ICC } \\ \text { Intraclass Correlation } & \text { L } \\ \text { Lateral } & \text { LV }\end{array}$

Left Ventricular blood volume LVV

LOA Level of agreement

Motion-mode M-Mode

Myocardial Infarction MI

Repetition Time $\quad$ TR

Septal S

Stroke Volume SV

Wall Motion Severity Index WMSI

\section{References}

1. Scherrer-Crosbie M, Thibault H. Echocardiography in Translational Research: Of Mice and Men. J Am Soc Echocardiogr. 200AD;21(10):1083-1092. doi:10.1016/j.echo.2008.07.001.Echocardiography

2. Gardin JM, Siri FM, Kitsis RN, Edwards JG, Leinwand LA. Echocardiographic Assessment of Left Ventricular Mass and Systolic Function in Micetle. Circ Res. 1995;76:9-7-914.

3. Rottman JN, Ni MDG, Brown M. IMAGING IN BASIC SCIENCE . MOUSE-ECHO: FROM BEDSIDE TO BENCH: PART II Echocardiographic Evaluation of Ventricular Function in Mice. 2007;24(1). doi:10.1111/j.1540-8175.2006.00356.x

4. Dawson D, Lygate CA, Saunders J, et al. Quantitative 3-Dimensional Echocardiography for Accurate and Rapid Cardiac Phenotype Characterization in Mice. 2004;108(suppl IV):1632-1637. doi:10.1161/01.CIR.0000142049.14227.AD 
5. Benavides-Vallve C, Corbacho D, Iglesias-Garcia O, et al. New strategies for echocardiographic evaluation of left ventricular function in a mouse model of long-term myocardial infarction. PLOS One. 2012;7(7):1-9. doi:10.1371/journal.pone.0041691

6. Parisi F, Moynihan PF, Feldman CL. Approaches to Determination of Left Ventricular Volume and Ejection Fraction by Real-Time Two-Dimensional Echocardiography. Clin Cardiol. 1979;2:257-263.

7. Lang RM, Badano LP, Mor-avi V, et al. Recommendations for Cardiac Chamber Quantification by Echocardiography in Adults: An Update from the American Society of Echocardiography and the European Association of Cardiovascular Imaging. J Am Soc Echocardiogr. 2015;28(1):1-39.e14. doi:10.1016/j.echo.2014.10.003

8. Lindsey ML, Kassiri Z, Virag JAI, de Castro Brás LE, Scherrer-Crosbie M. Guidelines for measuring cardiac physiology in mice. Am J Physiol Circ Physiol. 2018;314(4):H733-H752. doi:10.1152/ajpheart.00339.2017

9. Gao X, Dart AM, Dewar E, Jennings G, Du X. Serial echocardiographic assessment of left ventricular dimensions and function after myocardial infarction in mice. 2000;45:330-338.

10. Patten RD, Aronovitz MJ, Deras-mejia LUZ, et al. Ventricular remodeling in a mouse model of myocardial infarction. Am J Physiol - Hear Circ Physiol. 1998;274(5):1812-1820.

11. Lindsey ML, Kassiri Z, Virag JAI, de Castro Bras LE, Scherrer-Crosbie M. Guidelines for Measuring Cardiac Physiology in Mice. Am J Physiol Circ Physiol. 2018:ajpheart.00339.2017. doi:10.1152/ajpheart.00339.2017

12. Dorosz JL, Lezotte DC, Weitzenkamp DA, Allen LA SE. Performance of 3-Dimensional Echocardiography in Measuring Left Ventricular Volumes and Ejection Fraction A Systematic Review and Meta-Analysis. JACC. 2012;59(20):1799-1808. doi:10.1016/j.jacc.2012.01.037

13. Damen FW, Berman AG, Soepriatna AH, et al. High-Frequency 4-Dimensional Ultrasound (4DUS): A Reliable Method for Assessing Murine Cardiac Function. Tomography. 2017;3(4):180-187. doi:10.18383/j.tom.2017.00016

14. Soepriatna AH, Damen FW, Vlachos PP, Goergen CJ. Cardiac and Respiratory-Gated Volumetric Murine Ultrasound Arvin. Int J Cardiovasc Imaging. 2018;34(5):713-724. doi:10.1002/cncr.27633.Percutaneous

15. Grune J, Blumrich A, Brix S, et al. Evaluation of a commercial multi-dimensional echocardiography technique for ventricular volumetry in small animals. Cardiovasc Ultrasound. 2018;16(1):1-13. doi:10.1186/s12947-018-0128-9

16. Price AN, Cheung KK, Cleary JO, Campbell AE, Riegler J, Lythgoe MF. Cardiovascular Magnetic Resonance Imaging in Experimental Models. 2010;(Lv):278-292.

17. Russo I, Micotti E, Fumagalli F, et al. A novel echocardiographic method closely agrees with cardiac magnetic resonance in the assessment of left ventricular function in infarcted mice. Sci Rep. 2019;9(1):1-10. doi:10.1038/s41598-019-40393-0

18. Akki A, Gupta A, Weiss RG. Magnetic resonance imaging and spectroscopy of the murine cardiovascular system. Am J Hear Ciculation Physiol. 2013;304(5):633-648. 
19. Kanno S, Lerner DL, Schuessler RB, Betsuyaku T, Yamada KA, Saffitz JE. Echocardiographic Evaluation of Ventricular Remodeling in a Mouse Model of Myocardial Infarction. :601-609. doi:10.1067/mje.2002.117560

20. St MG, Sutton J, Sharpe N. Clinical Cardiology: New Frontiers Left Ventricular Remodeling After Myocardial Infarction Pathophysiology and Therapy. 2000:2981-2988.

21. Salto-Tellez M, Yung Lim S, El-Oakley RM, Tang TP, Almsherqi ZA LS. Myocardial infarction in the C57BL/6J mouse: A quantifiable and highly reproducible experimental model. Cardiovasc Physiol. 2004;13(2).

22. Scherrer-Crosbie M, Steudel W, Hunziker PR, Liel-Cohen N, Ullrich R, Zapol WM PM. Threedimensional echocardiographic assessment of left ventricular wall motion abnormalities in mouse myocardial infarction. J Am Soc Echocardiogr. 1999;12(10):834-840.

23. Rodrigues AC, Hataishi R, Ichinose F, Bloch K, Duremeaux G, Picard M S-CM. Relationship of systolic dysfunction to area at risk and infarction size after ischemia-reperfusion in mice. J Am Soc Echocardiogr. 2004;17(9):948-953.

24. Morgan EE, Faulx MD, McElfresh TA, et al. Validation of echocardiographic methods for assessing left ventricular dysfunction in rats with myocardial infarction. Am J Physiol Circ Physiol. 2004;287(5):H2049-H2053. doi:10.1152/ajpheart.00393.2004

25. Takagawa J, Zhang Y, Wong ML, et al. Myocardial infarct size measurement in the mouse chronic infarction model: comparison of area- and length-based approaches. J Appl Physiol. 2007;102(6):2104-2111. doi:10.1152/japplphysiol.00033.2007

26. Sharpe N. Ventricular remodeling following myocardial infarction. Am J Cardiol. 1992;70(10):20-26.

27. Mcgaffin KR, Witham WG, Yester KA, et al. Cardiac-specific leptin receptor deletion exacerbates ischaemic heart failure in mice. 2011:60-71. doi:10.1093/cvr/cvq288

\section{Figures}




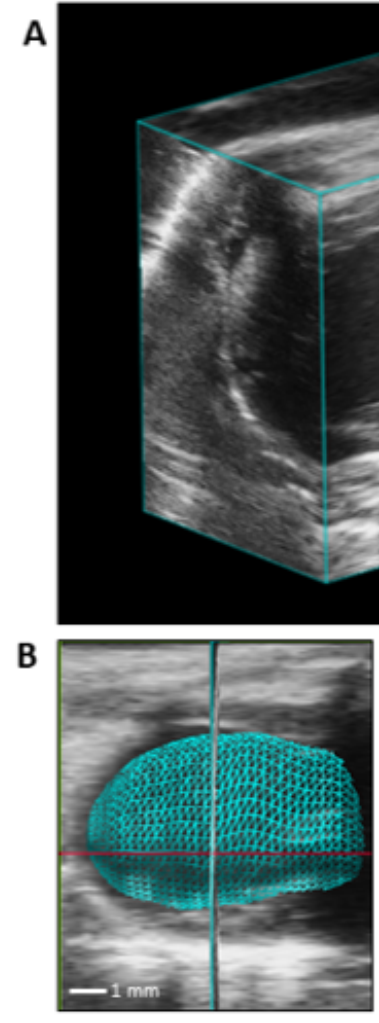

Diastole

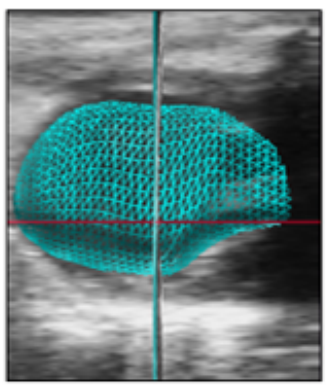

Systole
C
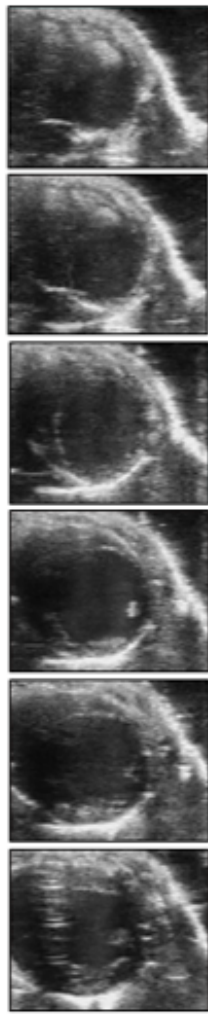

Diastole

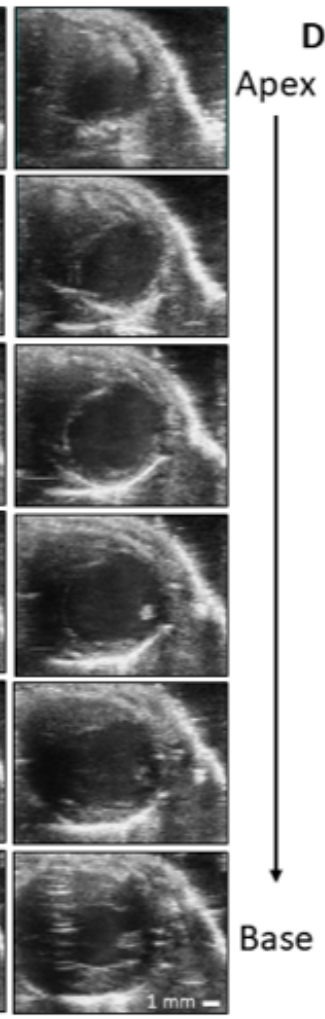

Systole

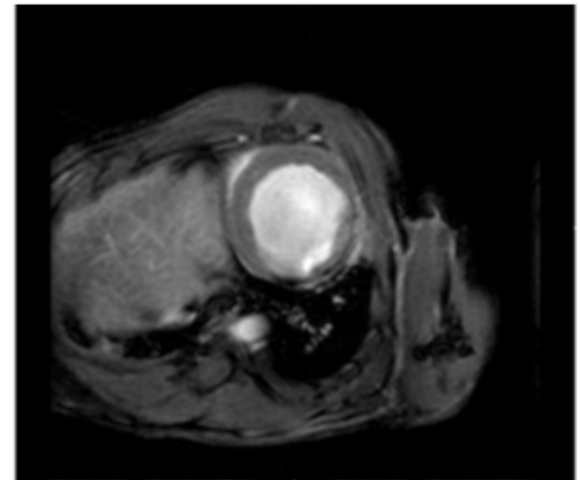

Diastole

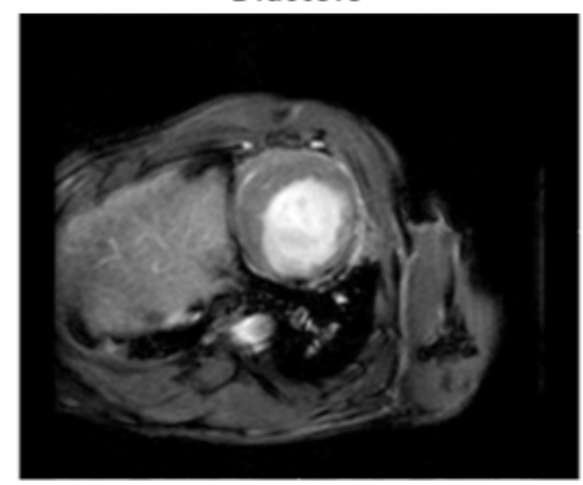

Systole

\section{Figure 1}

Representive Images Using 4D-US and CMR. A) 4D-US cube reconstruction of concentric stacked images in an infarcted heart. B) Representative wire tracing of 4D-US reconstruction in an infarcted heart 4 weeks after CAL. C) Representative short-axis images taken during end-diastole (left column) and end-systole (right column) at $1 \mathrm{~mm}$ intervals from the apex (top) to base (bottom) of an infarcted heart 4 weeks after CAL. D) Representative mid-ventricular CMR images of an infarcted heart during end-diastole (top) and end-systole (bottom). 
A

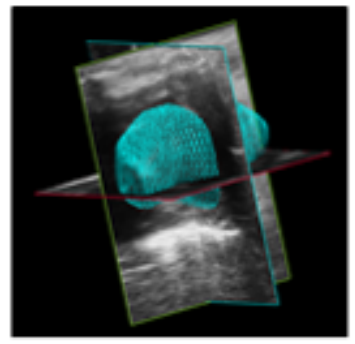

4D-US vs CMR

B
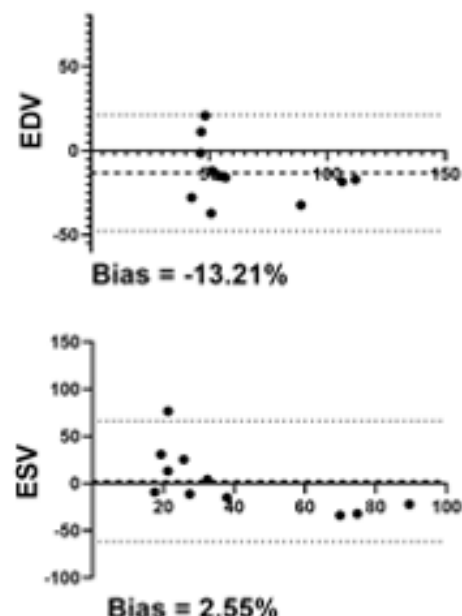

Bias $=2.55 \%$

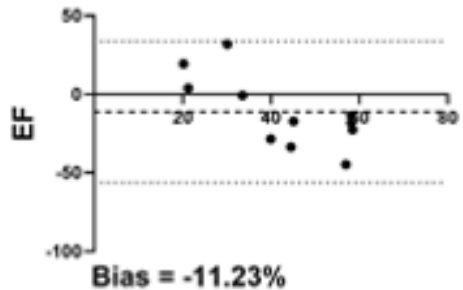

C

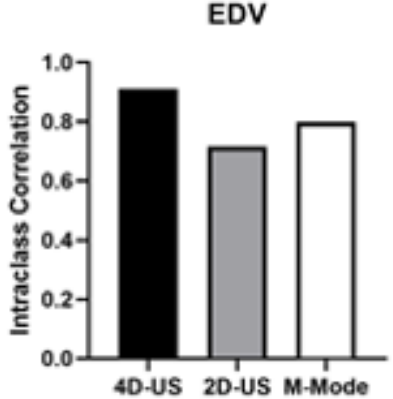

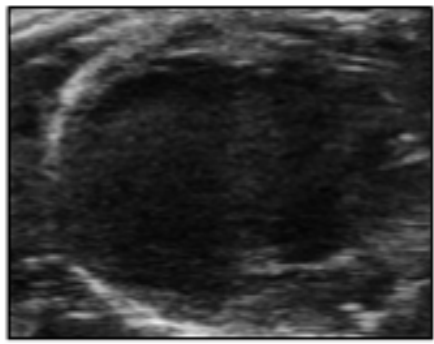

2D-US vs CMR
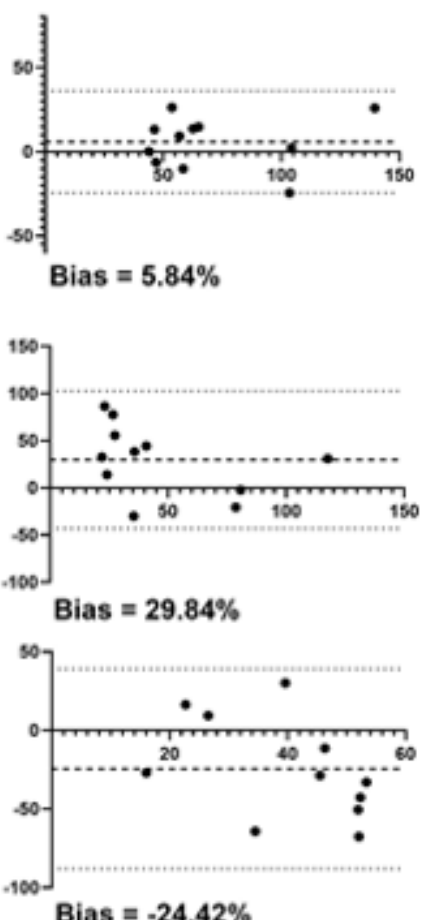

Bias $=-24.42 \%$

ESV

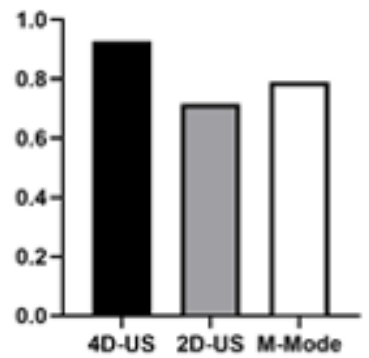

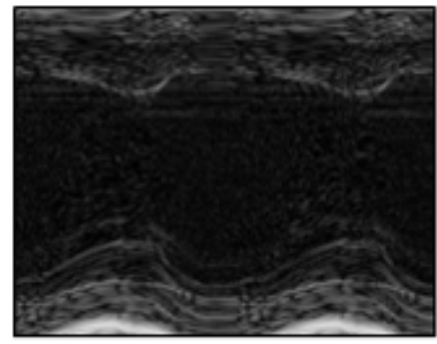

M-Mode vs CMR
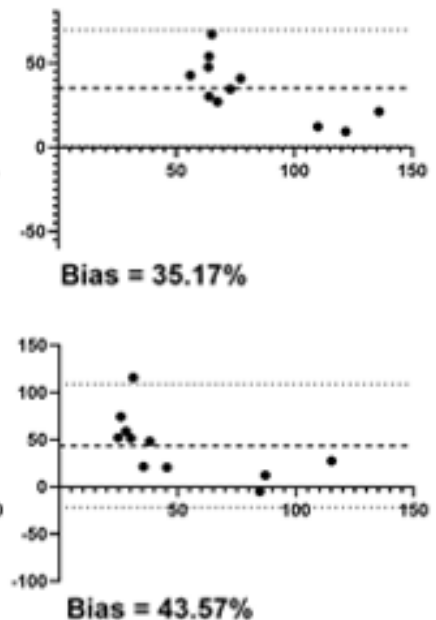

Bias $=43.57 \%$

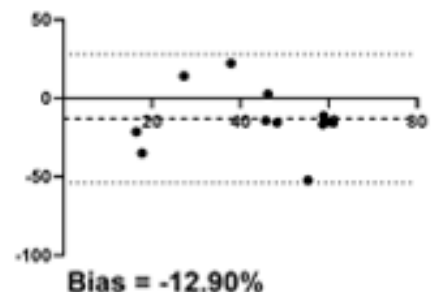

Bias $=-12.90 \%$

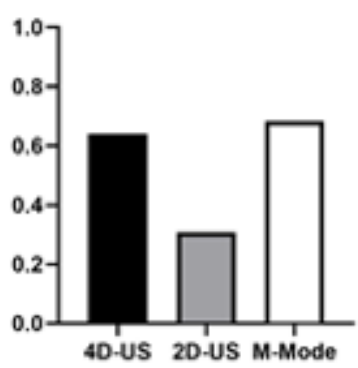

Figure 2

Bland Altman Analysis and Inter-user Variability of ECHO Modalities. A) Representative ECHO modalities for 4D-US, 2D-US, and M-Mode B) Bland-Altman analysis demonstrating the difference between selected measurement modality (4D-US, 2D-US, and M-Mode) and CMR. Data are presented as \% bias and dotted lines represent $95 \%$ confidence interval. $\mathrm{X}$-axes represent $\mathrm{CMR}$ volumetric measurements and $\mathrm{Y}$-axes represent \% Difference of ECHO values over CMR values. C) Inter-user variability between two users for each $\mathrm{ECHO}$ modality as calculated by Intraclass Correlation. 

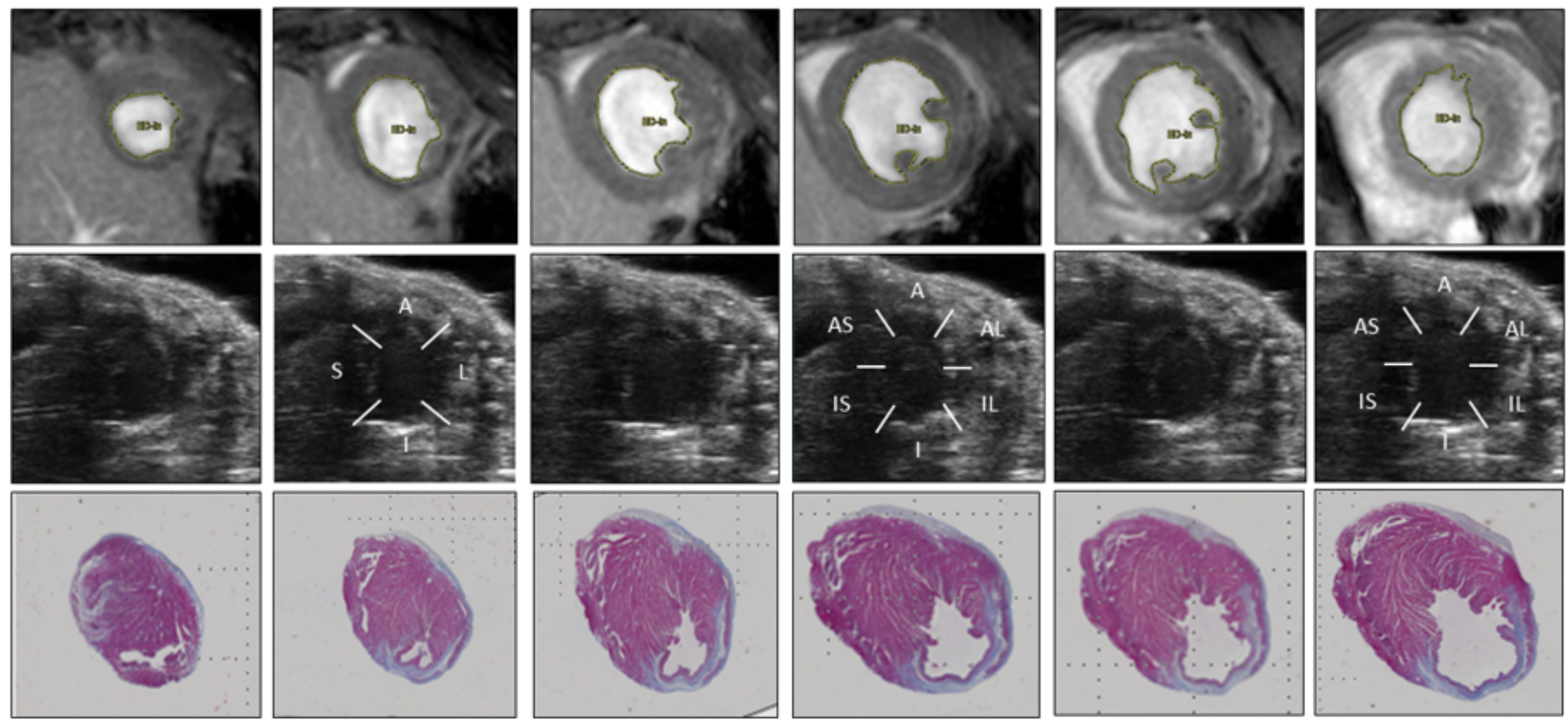

\section{Figure 3}

Representative Concentric Images of a Single Heart by CMR, 4D-US, and Histology. CMR (top row), 4D-US (middle row), and trichrome-stained histologic slides taken from a single infarcted heart at $1 \mathrm{~mm}$ steps from the apex (left) through basal-ventricle (right). 4D-US images (middle row) are divided into 16 sections for wall-motion score index, labeled as anterior (A), lateral ( $L)$, inferior (I), septal (S), anteriorlateral (AL), inferior-lateral (IL), inferior-septal (IS), and anterior-septal (AS).
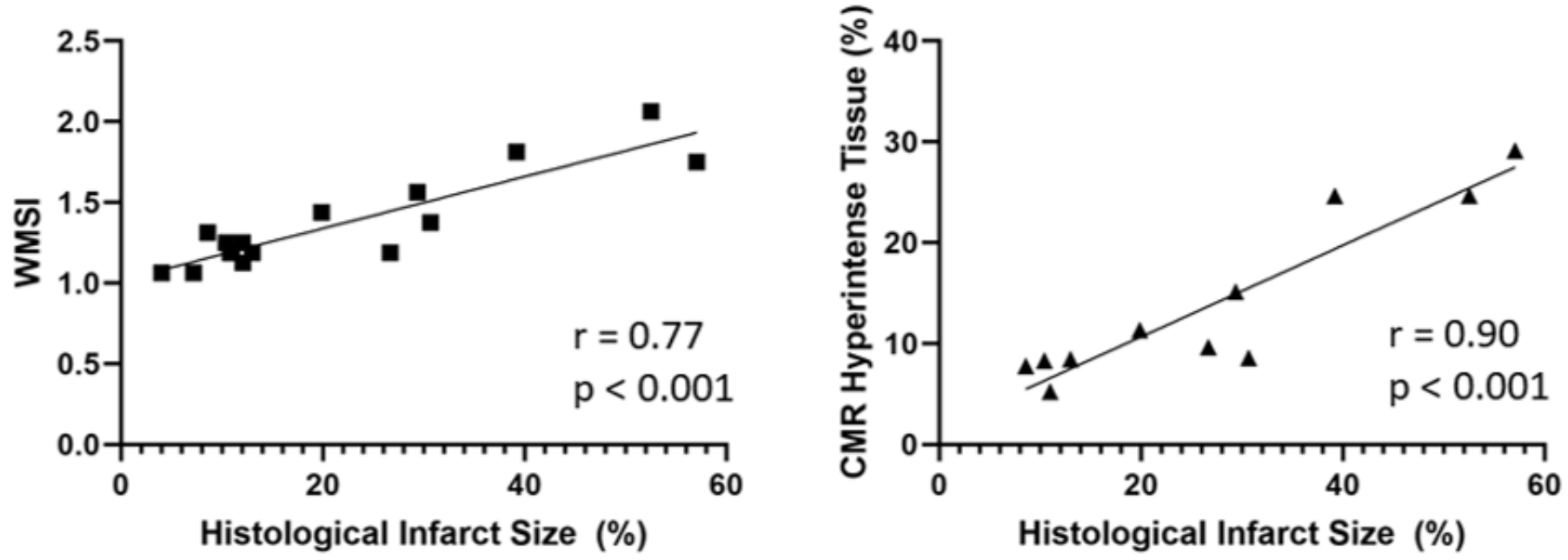

Figure 4

Correlation between WMSI and CMR to Histology. Regression analysis with Spearman rank correlation comparing wall motion score index (WMSI) to histological infarct size (left), and CMR hyperintense tissue to histologic infarct size (right). 

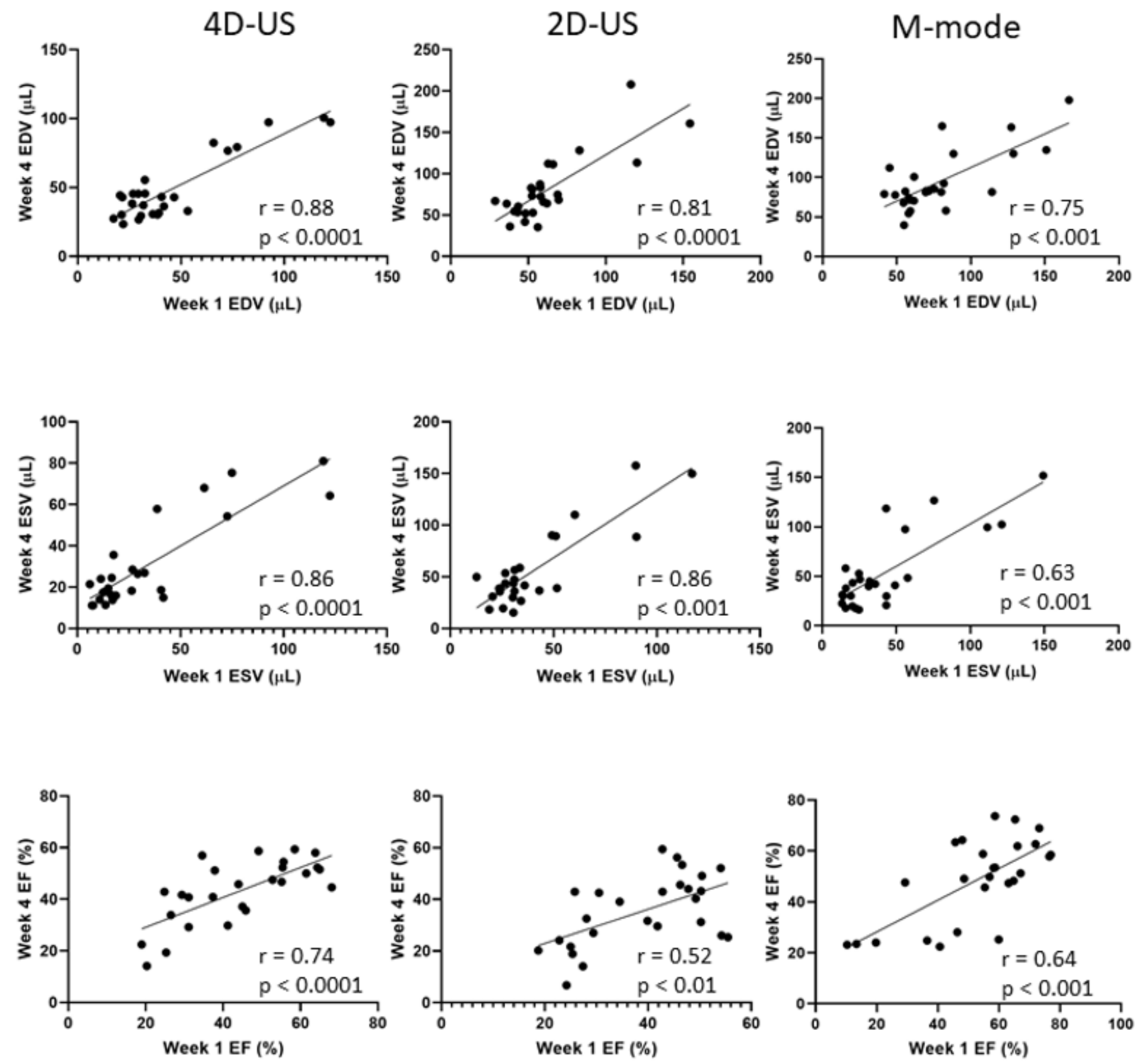

Figure 5

4D-US Demonstrates the Highest Correlation between Week 1 and Week 4 Volumetric Measurements.

Regression analysis with Pearson correlation comparing individual ECHO modalities taken at 1 week and 4 weeks following CAL.

\section{Supplementary Files}

This is a list of supplementary files associated with this preprint. Click to download. 
- AdditionalFile.docx

Page 20/20 